\title{
Joint-modal Distribution-based Similarity Hashing for Large-scale Unsupervised Deep Cross-modal Retrieval
}

\author{
Song Liu \\ School of Electronic and Computer \\ Engineering, Peking University \\ slpku@pku.edu.cn
}

\author{
Shengsheng Qian* \\ National Lab of Pattern Recognition, \\ Institute of Automation, Chinese \\ Academy of Sciences \\ shengsheng.qian@nlpr.ia.ac.cn
}

\author{
Yang Guan \\ iQIYI ltd \\ guanyanggy@163.com
}

\author{
Jiawei Zhan \\ School of Electronic and Computer \\ Engineering, Peking University \\ zhanjw@pku.edu.cn
}

\author{
Long Ying \\ School of Computer and Software, \\ Nanjing University of Information \\ Science Technology \\ lorin_ying@hotmail.com
}

\begin{abstract}
Hashing-based cross-modal search which aims to map multiple modality features into binary codes has attracted increasingly attention due to its storage and search efficiency especially in largescale database retrieval. Recent unsupervised deep cross-modal hashing methods have shown promising results. However, existing approaches typically suffer from two limitations: (1) They usually learn cross-modal similarity information separately or in a redundant fusion manner, which may fail to capture semantic correlations among instances from different modalities sufficiently and effectively. (2) They seldom consider the sampling and weighting schemes for unsupervised cross-modal hashing, resulting in the lack of satisfactory discriminative ability in hash codes.

To overcome these limitations, we propose a novel unsupervised deep cross-modal hashing method called Joint-modal Distributionbased Similarity Hashing (JDSH) for large-scale cross-modal retrieval. First, we propose a novel cross-modal joint-training method by constructing a joint-modal similarity matrix to fully preserve the cross-modal semantic correlations among instances. Second, we propose a sampling and weighting scheme termed the Distributionbased Similarity Decision and Weighting (DSDW) method for unsupervised cross-modal hashing, which is able to generate more discriminative hash codes by pushing semantic similar instance pairs closer and pulling semantic dissimilar instance pairs apart. The experimental results demonstrate the superiority of JDSH compared with several unsupervised cross-modal hashing methods on two public datasets NUS-WIDE and MIRFlickr. Our code is available at https://github.com/KaiserLew/JDSH.
\end{abstract}

\footnotetext{
${ }^{*}$ Corresponding author

Permission to make digital or hard copies of all or part of this work for personal or classroom use is granted without fee provided that copies are not made or distributed for profit or commercial advantage and that copies bear this notice and the full citation on the first page. Copyrights for components of this work owned by others than ACM must be honored. Abstracting with credit is permitted. To copy otherwise, or republish, to post on servers or to redistribute to lists, requires prior specific permission and/or a fee. Request permissions from permissions@acm.org.

SIGIR '2020, fuly 2020, Xi'an, China

(C) 2020 Association for Computing Machinery.

ACM ISBN 978-1-4503-XXXX-X/18/06 ..\$15.00

https://doi.org/10.1145/1122445.1122456
}

\section{CCS CONCEPTS}

- Information systems $\rightarrow$ Multimedia and multimodal retrieval; Nearest-neighbor search.

\section{KEYWORDS}

Large-scale multimedia retrieval, Cross-modal retrieval, Unsupervised cross-modal hashing

\section{INTRODUCTION}

Cross-modal similarity retrieval has been a popular research topic $[14,18,20,25,28,28,32,33,37,40]$ with the objective to search the semantic similar instances from different modalities. In a typical scenario, instances in one modality, e.g., images, are retrieved given a query from another modality, e.g., text. Hashing based cross-modal retrieval methods [14, 17, 18, 20, 25, 32, 33, 41] largely improve the retrieval efficiency on both speed and storage by mapping large-scale and high-dimensional multi-modal media data into a common Hamming space, where semantic similar instances from different modalities can be represented by similar hash codes and the correlations among these instances can be effectively measured with their Hamming distance. Since different modality instances are heterogeneous in terms of their feature representation and distribution, it is necessary for hashing based cross-modal retrieval methods to explore appropriate methodologies that the modality gap can be bridged.

Conventional cross-modal hashing methods can be divided into unsupervised and supervised methods. For the supervised methods [1, 2, 8, 14, 18, 19, 34, 36, 39], manually-annotated labels, requiring expensive human cost and computation, are leveraged to construct the similarity matrices and supervise hash code generation. Unsupervised cross-modal hashing methods [5, 25, 33] have been broadly discussed because of the substantial reduction of computation cost and more widely application. This paper will focus on the learning of unsupervised cross-modal hashing.

As deep neural networks $[10,12,16,23,26,27]$ achieve outstanding performance on many classic scenarios and can be used as nonlinear hash functions to enable end-to-end learning of deep representation and hash codes, many unsupervised deep cross-modal hashing methods have been proposed to obtain semantic similar 
information by leveraging features extracted from the pre-trained deep networks. Recently, UDCMH [33] is proposed to learn hash functions for image and text modalities by implementing the matrix factorization and the Laplacian constraints with the intention to preserve correlations among instances. However, one major limitation of UDCMH is that it only preserves neighborhood structures of original data from multiple modalities separately, and cannot utilize multiple modality similarity information to complement each other. Though DJSRH [25] proposes a joint-semantics similarity matrix to integrate the multiple modality similarity information for cross-modal instances, simultaneously. However, the integration manner adopted by DJSRH is redundant and suboptimal so that much unimportant information is concerned. For example, we can construct an image modality similarity matrix $\mathbf{S}_{v}$ by adopting image pre-trained features and construct a text modality similarity matrix $\mathbf{S}_{t}$ by adopting text pre-trained features. The intra-modal similarity information is fully preserved in $\mathbf{S}_{v}$ and $\mathbf{S}_{t}$. However, the similarity matrix in DJSRH includes the intra-modal fusion items $\mathbf{S}_{v} \mathbf{S}_{v}^{\mathrm{T}}$ and $\mathbf{S}_{t} \mathbf{S}_{t}^{\mathrm{T}}$ which deviate from the purpose of fusing multiple modality similarity information, and bring redundant information.

Another drawback of existing unsupervised deep cross-modal hashing methods is that they directly generate hash codes by preserving the correlations among the continuous pre-trained features in the Hamming space that may cause hash codes lack of the better discriminative ability. In reality, in the supervised setting, the supervised information is annotated by multiple labels and we define the similarity signal $s_{i j}=1$ if two instances share as least one label, otherwise $s_{i j}=0$. In the unsupervised setting, the similarity signal $s_{i j} \in \mathbb{R}$ and it results that generated hash codes lack of better distinguishing ability compared with supervised setting. For example, the Hamming distance among one instance and other similar instances could not be much larger than that among other dissimilar instances. Meanwhile, plenty of methods have been proposed recently to improve sampling and weighting schemes that focus on pulling semantic similar instance pairs closer, pushing semantic dissimilar instance pairs apart from each other for supervised pairbased problems, such as contrastive loss [9], triplet loss [11], ms loss [30]. Although pair-based sampling and weighting schemes have been successfully used for the supervised cross-modal hashing learning to learn discriminative feature representations and hash codes, to the best of our knowledge, no existing work has explored to handle unsupervised cross-modal hashing learning.

In order to promote the performance of unsupervised hashingbased cross-modal retrieval, we need to address the two following challenges:

- Challenge 1: How to effectively fuse multiple modality similarity information jointly that semantic correlations among instances can be fully preserved in hash codes? Existing methods usually learn multiple modality similarity information separately or in a redundant fusion manner, which may cause hash functions fail to fully and properly capture essential semantic correlations among instances.

- Challenge 2: How to enhance the discriminative ability of hash codes and improve sampling and weighting schemes for unsupervised cross-modal hashing? Existing methods directly generate hash codes by preserving the correlations
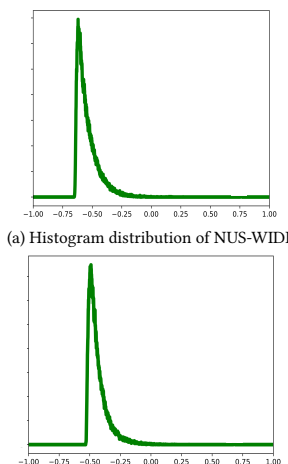

(d) Histogram distribution of MIRFlick (a) Histogram distribution of NUS-WIDE
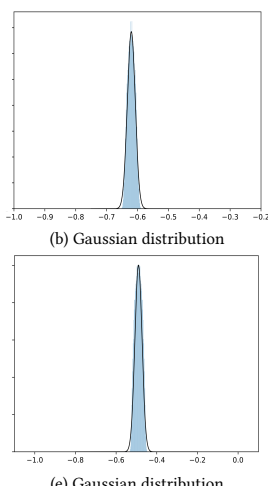

(e) Gaussian distribution (b) Gaussian distributio

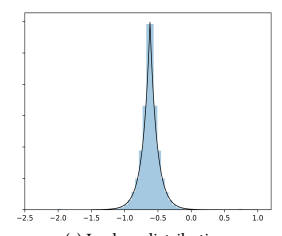

(c) Laplace distribution

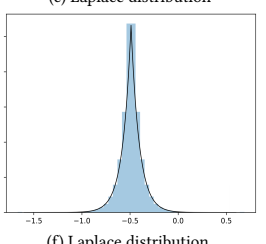

(f) Laplace distribution
Figure 1: Statistics of S. (a) Similarity histogram distribution of NUS-WIDE. (b) Gaussian estimation for the left part of (a). (c) Laplace estimation for the right part of (a). (d) Similarity histogram distribution of MIRFlickr. (e) Gaussian estimation for the left part of (d). (f) Laplace estimation for the right part of (d).

among the continuous pre-trained features in the Hamming space, which may cause hash codes lack of the satisfactory discriminative ability.

To overcome the above challenges, we propose a novel unsupervised deep cross-modal hashing method termed Joint-modal Distribution-based Similarity Hashing (JDSH) for large-scale crossmodal retrieval. For Challenge 1, we first propose a novel crossmodal joint-training method by fusing $\mathbf{S}_{v}$ and $\mathbf{S}_{t}$ in a unified matrix $\mathbf{S}$, which can sufficiently preserve cross-modal neighborhood relations among instances and fully mine the latent modality information to bridge the modality gap better. For Challenge 2, we propose the Distribution-based Similarity Decision and Weighting (DSDW) method to learn more discriminative hash codes in the unsupervised setting. First, we define four types of similarities to exactly measure the similarity among instance pairs in unsupervised pair-based problems: self-similarity, relative similarity, relative dissimilarity and extreme dissimilarity. Then, we manage to push semantic similar instance pairs closer and pull semantic dissimilar instance pairs apart. However, except for self-similarity, there is no similarity type decision for an instance pair in the unsupervised setting. To solve this problem, we analyze the statistics of $\mathbf{S}$ which are shown in Figure 1 and set two similarity thresholds $s_{r}$ and $s_{l}$ according to the similarity distribution estimation. Afterward, we impose the designed weighting scheme to instances that can push instance pairs with $s>s_{r}$ closer and pull instance pairs with $s<s_{l}$ apart.

In brief, the contributions of this paper are listed as follows:

- We propose a novel Joint-modal Distribution-based Similarity Hashing (JDSH) for large-scale unsupervised cross-modal retrieval by constructing a joint-modal similarity matrix for fusing cross-modal similarity information and proposing the Distribution-based Similarity Decision and Weighting method for improving the sampling and weighting schemes of unsupervised cross-modal hashing.

- We propose a novel cross-modal joint-training method by constructing a joint-modal similarity matrix used to supervise hash code generation, which can make hash codes have 
the high preservation of original semantic correlations among the instances.

- We propose the Distribution-based Similarity Decision and Weighting (DSDW) method by setting two similarity thresholds, pushing similar instance pairs closer and pulling dissimilar instance pairs apart from each other accordingly, which can make the generated hash codes have better discriminative ability.

- Extensive experiments demonstrate the superiority of JDSH compared with seven unsupervised shadow and deep crossmodal hashing methods on two datasets NUS-WIDE and MIRFlickr.

\section{RELATED WORK}

Recently, unsupervised cross-modal hashing methods have been widely discussed because of the efficient computation and accurate retrieval. In this section, we will briefly introduce some representative unsupervised shadow and deep cross-modal hashing methods.

Unsupervised shadow cross-modal hashing. As two of the earliest unsupervised cross-modal hashing methods, both Crossview hashing (CVH) [17] and Inter-Media Hashing (IMH) [24] extend Spectral Hashing [31] from unimodal hashing to cross-modal hashing. They learn hash function by solving eigenvalue decomposition with constructed similarity graph. Collective Matrix Factorization Hashing (CMFH) [6] utilizes matrix factorization methods to learn hash functions that bridge the modality gap. Latent Semantic Sparse Hashing (LSSH) [41] updates CMFH by learning latent features from images and texts jointly with sparse coding in matrix factorization to learn hash codes. However, most of these methods are built based on shallow architectures, and are difficult to effectively capture the cross-modal heterogeneous relationships by utilizing hand-crafted features.

Unsupervised deep cross-modal hashing. As deep neural networks (DNN) have achieved extraordinary performances on nonlinear representation learning, many unsupervised deep crossmodal hashing methods utilize deep schemes to learn hash functions, and obtain superior performances in many cross-modal retrieval tasks. There are some representative methods need to be noticed. For solving the optimization of concrete constraint problem in cross-modal hashing methods, DBRC [5] utilizes the adaptive tanh function which is easy to directly optimize the objective function and generates the binary hash codes in an unsupervised manner without relaxing the concrete constraint and performing thresholding strategy. UDCMH [33] implements the matrix factorization in unsupervised deep cross-modal hashing and incorporates the graph Laplacian constraint into the objective function which preserve neighborhood structures of original data from multiple modalities separately. DJSRH [25] proposes a joint-semantics similarity matrix for cross-modal instances which integrates the multiple modality similarity information simultaneously and a hash code reconstructing framework that maximally reconstruct joint-semantics relations in Hamming space.

Although these methods have achieved breakthrough, there are still some challenges to be solved that are mentioned above. In this paper, we focus on improving the performance of unsupervised deep cross-modal hashing in two aspects: (1) to effectively utilize multiple modality similarity information with the intention to fully preserve similarity information in hash codes and better bridge modality gap in Hamming space. (2) to improve the sampling and weighting schemes for unsupervised deep cross-modal hashing methods and generate more discriminative hash codes for cross modalities.

\section{NOTATION AND PROBLEM DEFINITION}

We firstly introduce some notations that are used in this paper. Assuming that the training set $\mathbf{O}=\left\{v_{i}, t_{i}\right\}_{i=1}^{N}$ contains $n$ imagetext pairs. $v_{i} \in \mathbb{R}^{d_{v}}$ and $t_{i} \in \mathbb{R}^{d_{t}}$ are the image and text features of the $i$-th instance, where $d_{v}$ and $d_{t}$ represent the dimensions of image and text features. We use $\mathbf{V}=\left\{v_{i}\right\}_{i=1}^{N}$ and $\mathbf{T}=\left\{t_{i}\right\}_{i=1}^{N}$ to represent image-feature and text-feature matrix respectively.

Given $\mathbf{V}$ and $\mathbf{T}$, JDSH aims to learn hash functions $f_{v}\left(v ; \theta_{v}\right)$ and $f_{t}\left(t ; \theta_{t}\right)$, and simultaneously generates hash code $\mathbf{B}_{v} \in\{-1,+1\}^{N \times K}$ and $\mathbf{B}_{t} \in\{-1,+1\}^{N \times K}$ for image and text modalities respectively, where $K$ is the length of the hash code, $\theta_{v}, \theta_{t}$ represent the parameters of ImgNet for image feature learning and TextNet for text feature learning respectively. We denote $m$ to the mini-batch size. Semantic similar image item $v_{i}$ and text item $t_{j}$ should be represented by similar hash code $\mathbf{B}_{v}^{i}$ and $\mathbf{B}_{t}^{j}$.

\section{METHODOLOGY}

\subsection{Network Architecture}

Following [5, 25, 33], we adopt AlexNet [16] and Multi-Layer Perceptions (MLP) [22] as the backbone of the ImgNet and the TextNet, which are used to the feature learning of image and text modalities respectively. The inputs of ImgNet are the raw images and the inputs of TextNet are the topic vectors or the tag occurrence features provided by datasets.

ImgNet. Specifically, the original AlexNet model contains five convolution layers and three fully-connected layers $\left(f c_{v 6}-f c_{v 8}\right)$. We replace the classification layer $f c_{v 8}$ with a new hashing layer $f c_{h}$. The $f c_{h}$ has $K$ hidden units and outputs continuous features $\mathbf{F}_{v} \in \mathbb{R}^{N \times K}$. The $f c_{v 7}$ outputs image features $\mathbf{V}=\left\{v_{i}\right\}_{i=1}^{N}$, where $v_{i} \in \mathbb{R}^{d_{v}}, d_{v}=4096$. Later we will utilize $\mathbf{V}$ to construct the image modality similarity matrix $\mathbf{S}_{v}$.

TextNet. MLP consists of two fully-connected layers $f c_{t 1}$ and $f c_{t 2}\left(d_{t} \rightarrow 4096 \rightarrow K\right)$ and generates continuous features $\mathbf{F}_{t} \in$ $\mathbb{R}^{N \times K}$. Later we will directly utilize the inputs of TextNet as $\mathbf{T}=$ $\left\{t_{i}\right\}_{i=1}^{N}$ where $t_{i} \in \mathbb{R}^{d_{t}}$ to construct the text modality similarity matrix $\mathbf{S}_{t}$.

\subsection{Joint-modal Similarity Matrix Construction}

In unsupervised cross-modal hashing methods, the multi-label annotation of instances is not available, we cannot construct the pairwise multi-label similarity matrix that is used to describe semantic similarities. To obtain semantic structures without multi-label annotation, $[5,7,25,33,35]$ show that features extracted from pre-trained deep neural networks contain abundant semantic information such that we can leverage the pre-trained features $\mathbf{V}=\left\{v_{i}\right\}_{i=1}^{N}$ to construct the image modality cosine similarity matrix $\mathbf{S}_{v}=\left\{s_{i j}^{v}\right\}_{i, j=1}^{N}$ 


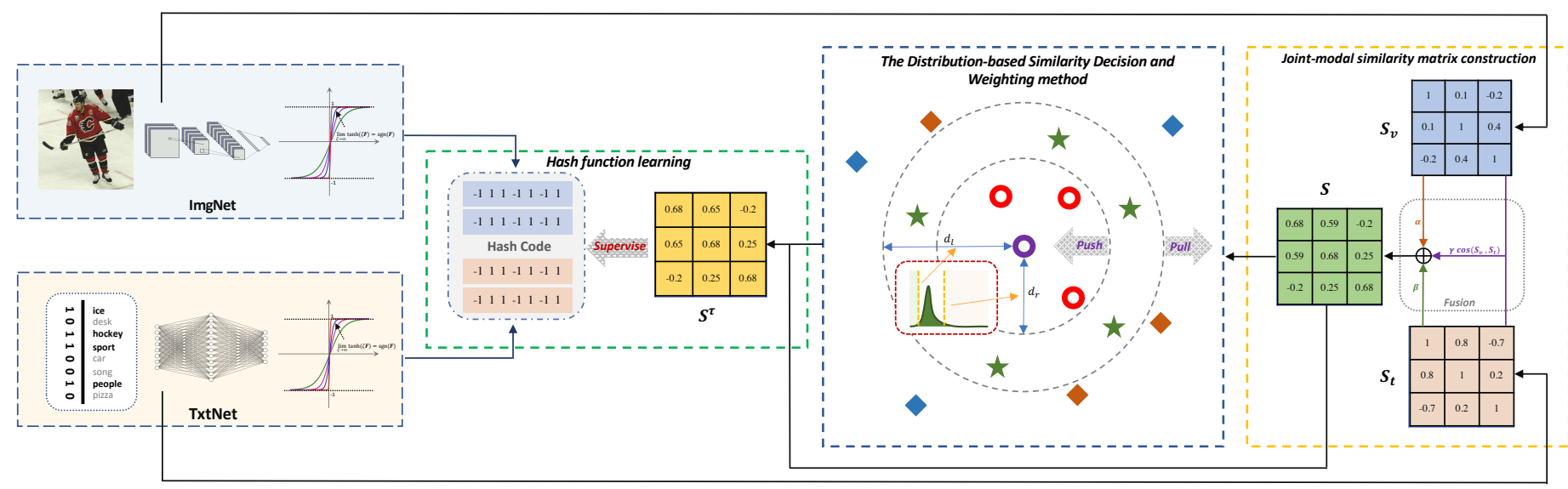

Figure 2: The pipeline of JDSH: (1) Learn features and generate hash codes for image and text modality instances; (2) Utilize pre-trained features to construct image modality cosine similarity matrix $S_{v}$ and text modality cosine similarity matrix $S_{t}$; (3) Joint-modal similarity matrix $S$ construction; (4) Refine $S$ by the Distribution-based Similarity Decision and Weighting (DSDW) method and obtain $S^{\tau}$; (4) Hash function learning for image and text modalities.

where $s_{i j}^{v}$ is defined as $\cos \left(v_{i}, v_{j}\right)=\frac{v_{i} v_{j}^{\mathrm{T}}}{\left\|v_{i}\right\|_{2}\left\|v_{j}\right\|_{2}} \in[-1,+1]$ that represents the cosine similarity of image pre-trained features $v_{i}, v_{j} \in$ $\mathbb{R}^{d_{v}}$.

To construct the text modality similarity matrix $\mathbf{S}_{t}=\left\{s_{i j}^{t}\right\}_{i, j=1}^{N}$, we directly adopt the inputs of TextNet as text features $\mathbf{T}=\left\{t_{i}\right\}_{i=1}^{N}$, $t_{i} \in \mathbb{R}^{d_{t}}$, where each of $t_{i}$ includes some textual tags corresponding to content description of an image-text pair. $s_{i j}^{t}$ is defined as $\cos \left(t_{i}, t_{j}\right)=\frac{t_{i} t_{j}^{\mathrm{T}}}{\left\|t_{i}\right\|_{2}\left\|t_{j}\right\|_{2}} \in[-1,+1]$.

Then we need to fuse the image modality similarity matrix $\mathbf{S}_{v}$ and text modality similarity matrix $\mathbf{S}_{t}$ in a unified similarity matrix which can both preserve semantic relations among instances from different modalities and complement to each other. To this end, we propose a joint-modal similarity matrix $\mathbf{S}$ to fuse cross-modal similarity information in $\mathbf{S}_{v}$ and $\mathbf{S}_{t}$.

$$
\begin{aligned}
& \quad \mathbf{S}=\alpha \mathbf{S}_{v}+\beta \mathbf{S}_{t}+\gamma \cos \left(\mathbf{S}_{v}, \mathbf{S}_{t}\right) \\
& \quad=\left\{s_{i j}\right\}_{i, j=1}^{N} \\
& \text { s.t. } \alpha, \beta, \gamma \geq 0, \alpha+\beta+\gamma=1 \\
& \quad s_{i j} \in[-1,1]
\end{aligned}
$$

where $s_{i j}$ is the pairwise similarity of an image-text pair that refines the cosine similarity relations from both image and text modalities. $\alpha, \beta, \gamma$ are the trade-off parameters that control the importance of similarity information from different modalities. An image-text pair with larger $s_{i j}$ is obvious more semantic similar than the others with smaller $s_{i j}$.

We calculate $\cos \left(\mathbf{S}_{v}, \mathbf{S}_{t}\right)$ based on the assumption that two semantic similar instances in one modality should obtain the similar similarity relations with other instances in another modality. Eq.(1) combines the similarity information from different modalities sufficiently in a unified matrix which can fully mine the latent modality intrinsic properties and complement neighborhood relations to each other from two modalities.

\subsection{The Distribution-based Similarity Decision and Weighting}

4.3.1 Multiple Similarities. Wang et al. [30] defines three types of similarities and analyzes several supervised pair-based loss functions $[9,11,21,29,30,38]$ according to whether they fully consider different types of similarities that are valuable for us to understand deep supervised metric learning comprehensively. However, in the unsupervised setting, this issue has not received widely attention. By extending [30], we define and analyze four types of similarities for unsupervised pair-based problems: self-similarity, relative similarity, relative dissimilarity and extreme dissimilarity.

Self-similarity (SS). A self-similarity is calculated by one instance itself through cosine similarity calculation or other similarity calculation methods, and is the largest similarity value one instance can obtain. The similarity matrix $\mathbf{S}=\left\{s_{i j}\right\}_{i, j=1}^{N}$ contains $N$ self-similarity items $s_{i j}$, s.t. $i=j$ in the diagonal positions of the matrix. We assume that any pairs whose $s_{i j}$ approximating to the self-similarity refer to semantic similar pairs.

Extreme dissimilarity (ED). Extreme dissimilarity refers to the similarity between one instance and its extremely dissimilar instances. It is the minimum similarity value which one instance can obtain. In the distance metric space, the positions of instances with extreme dissimilarity are the farthest positions one instance can reach to. We assume that any pairs whose $s_{i j}$ approximating to the extreme dissimilarity refer to extremely semantic dissimilar pairs.

Relative similarity (RS). If the similarity between two instances is relative close to their self-similarity and is relative far from their extreme dissimilarity, we will consider them as a relative semantic similar instance pair and their relationship will be represented by relative dissimilarity. The relative semantic similar pairs are informative and meaningful for us to learn common features from them 

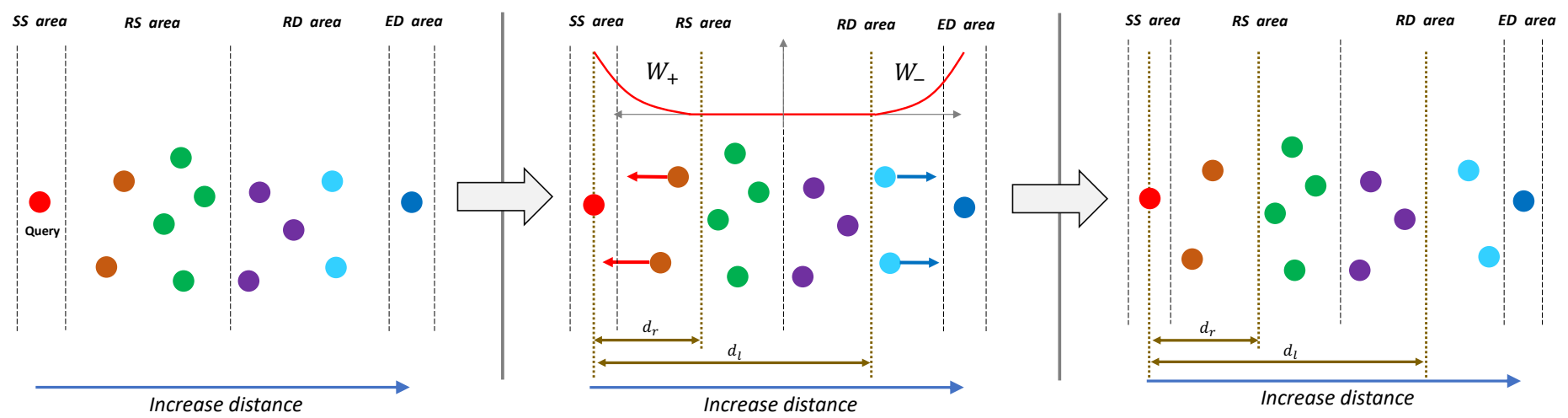

Figure 3: The illustration of the Distribution-based Similarity Decision and Weighting method (DSDW). (left): In the distance metric space, a query is located in SS area and the direction of $S S \rightarrow R S \rightarrow R D \rightarrow E D$ refers that the distance between the query and other instances increases; (middle): We set two thresholds $d_{r}$ and $d_{l}$; (right): Push the instances with $d<d_{r}$ closer to the SS area that can increase the similarity between the query and the corresponding instances by weighting $W_{+}$and pull the instances with $d>d_{l}$ closer to the ED area that can decrease the similarity between the query and the corresponding instances by weighting $W_{-}$.

in the feature learning process. Note that semantic similar pairs belong to relative semantic similar pairs.

Relative dissimilarity (RD). If the similarity between two instances is relative close to their extremely similarity and is relative far from their self-similarity, we will consider them as a relative dissimilar pair and their relationship will be represented by relative dissimilarity. The relative dissimilar pairs are significant and useful for us to learn more discriminative features in the feature learning process. Note that extremely semantic dissimilar pairs belong to relative semantic dissimilar pairs.

As mentioned above, the definition of four types of similarities is valuable for us to deeply investigate the relations among instances in unsupervised cross-modal hashing and other unsupervised pairbased problems. Thus, for the better generation of discriminative hash codes and making similar instance pairs closer and dissimilar instance pairs apart from each other, we will utilize the characteristics of similarities to further refine the similarity matrix $\mathbf{S}$. As shown in Figure 3 (left), in the distance metric space, we divide up four areas: SS area, RS area, RD area and ED area. The query is located in SS area and the direction of $S S \rightarrow R S \rightarrow R D \rightarrow E D$ refers that the distance between the query and other instances increases and the similarity decreases in the order of self-similarity, relative similarity, relative dissimilarity and extreme dissimilarity. In the Figure 3 (middle), we set two thresholds $d_{r}$ and $d_{l}$ that divide up the RS area and the RD area, respectively. Then we will decrease the distance between the query and the instances located in the left part of the RS area $\left(d<d_{r}\right)$ by pushing the corresponding instances closer to the SS area. Meanwhile, we will increase the distance between the query and the instances located in the right part of the RD area $\left(d>d_{l}\right)$ by pulling the corresponding instances closer to the ED area. By this way, We push similar instances closer and pull dissimilar instances apart from each other. Now, we raise the key problems: Where should we set the two thresholds? How should we push and pull instances?
4.3.2 Threshold Setting and Similarity Weighting. We random select 5,000 instance pairs from dataset NUS-WIDE and MIRFlickr and analyze the statistics of $\mathbf{S}=\left\{s_{i j}\right\}_{i, j=1}^{N}$ for all batches in one epoch (except for self-similarity). Figure 1(a) and (d) show the corresponding similarity histogram distribution for NUS-WIDE and MIRFlikcr. We find that the left part of similarity histogram distribution can be fit with a half Gaussian distribution and the right part can be fit with a half Laplace distribution.

Based on the above statistical characteristics and the definition of similarities, we propose a sampling and weighting scheme termed the Distribution-based Similarity Decision and Weighting (DSDW) method to refine $\mathbf{S}$. We split each similarity histogram distribution into two parts based on the maximum value and fit the left, right part with Gaussian distribution, Laplace distribution, respectively. The estimated results are shown in Figure 1(b), (c), (e), (f). They clearly show that the similarity histogram distribution can be fit with a left half Gaussian distribution and a right half Laplace distribution approximately. It should be noted that the estimations of two halves for similarity histogram distribution are not extremely standard Gaussian and Laplace distribution. However, we can intuitively understand the similarity distribution by the distribution estimation results and provide reference for setting $d_{l}$ and $d_{r}$.

Afterwards, we use $\mu_{l}$ and $\sigma_{l}$ to denote the mean and standard deviation of the left part Gaussian distribution and use $\mu_{r}$ and $\sigma_{r}$ for the right part Laplace distribution. Further, we set two similarity thresholds $s_{l}=\left(\mu_{l}-\eta_{l} \sigma_{l}\right)$ and $s_{r}=\left(\mu_{r}+\eta_{r} \sigma_{r}\right)$. If $s_{i j}$ for an instance pair is smaller than $s_{l}$, we will consider it as an extremely semantic dissimilar pair and set $s_{i j}$ closer to the extreme dissimilarity $s_{\text {min }}=$ $\min (\mathbf{S})$ by weighting $W_{-}$that can pull two instances apart. While if $s_{i j}$ for an instance pair is larger than $s_{r}$, we will consider it as an semantic similar pair and set $s_{i j}$ closer to the self-similarity $s_{\max }=\max (\mathbf{S})$ by weighting $W_{+}$that can pull two instances closer. $\eta_{l}$ and $\eta_{r}$ are two hyper-parameters to control the value of $s_{l}$ and $s_{r}$. 
Then we define $W_{+}$for the similarity $s_{i j}$ when $s_{\max }>s_{i j}>s_{r}$ $W_{+}$goes up exponentially with the increase of $s_{i j}$.

$$
W_{+}=1+\lambda_{1} e^{s_{i j}-s_{\text {max }}}
$$

We define $W_{-}$for the similarity $s_{i j}$ when $s_{\text {min }}<s_{i j}<s_{l}$. $W_{-}$ goes up exponentially with the decrease of $s_{i j}$.

$$
W_{-}=1+\lambda_{2} e^{s_{\text {min }}-s_{i j}}
$$

$W_{+}$and $W_{-}$are helpful for the hash functions to learn common features among similar instances and discriminative features among dissimilar instances, which can generate more discriminative and accurate hash codes. Finally, $\mathbf{S}$ is refined by this formulation:

$$
s_{i j}^{\tau}= \begin{cases}W_{+} s_{i j}, & s_{\max }>s_{i j}>s_{r} \\ W_{-} s_{i j}, & s_{\min }<s_{i j}<s_{l} \\ s_{i j}, & \text { others }\end{cases}
$$

So that we can obtain $\mathbf{S}^{\tau}=\left\{s_{i j}^{\tau}\right\}_{i, j=1}^{N} . s_{l}$ and $s_{r}$ are two similarity thresholds while $d_{l}$ and $d_{r}$ are two distance thresholds. According to the definition of cosine distance, we obtain $d_{r}=1-s_{r}$ and $d_{l}=1-s_{l}$

\subsection{Objective Function}

We can learn hash codes via minimizing the reconstruction error between the similarity matrix $\mathbf{S}^{\tau}$ and the inter- and intra-modal reconstruction of the to-be-learned hash codes $\mathbf{B}_{v}$ and $\mathbf{B}_{t}$ with the following objective function:

$$
\begin{gathered}
\min _{\mathbf{B}_{v}, \mathbf{B}_{t}}\left\|\varphi \mathbf{S}^{\tau}-\cos \left(\mathbf{B}_{v}, \mathbf{B}_{t}\right)\right\|_{F}^{2}+\rho_{1}\left\|\varphi \mathbf{S}^{\tau}-\cos \left(\mathbf{B}_{v}, \mathbf{B}_{v}\right)\right\|_{F}^{2} \\
+\rho_{2}\left\|\varphi \mathbf{S}^{\tau}-\cos \left(\mathbf{B}_{t}, \mathbf{B}_{t}\right)\right\|_{F}^{2} \\
\text { s.t. } \mathbf{S}^{\tau}=\left\{s_{i j}^{\tau}\right\}_{i, j=1}^{N} \in[-1,+1]^{N \times N}, \\
\mathbf{B}_{v}, \mathbf{B}_{t} \in\{-1,+1\}^{N \times K}
\end{gathered}
$$

where $\rho_{1}$ and $\rho_{2}$ are the trade-off parameters to balance the interand intra-modal reconstruction. $\varphi$ is the scale parameter which makes the reconstruction more flexible.

\subsection{Optimization}

To obtain hash codes $\mathbf{B}_{v}$ and $\mathbf{B}_{t}$, a sign activation function $\mathbf{B}=$ $\operatorname{sgn}(\mathbf{F})$ is needed to binarize the $K$-dimensional continues representation $\mathbf{F}$ into K-bit hash codes $\mathbf{B} \in\{-1,+1\}^{N \times K}$, where $\mathbf{F} \in \mathbb{R}^{N \times K}$ is the output of the last layer of the deep hashing network before activation. However, because of the sign function's non-smooth and non-convex, if we adopt sign as the activation function within deep networks, all nonzero inputs will cause zero gradient, which is infeasible for training deep networks. This is known as vanishing gradient problem. Following [3, 5, 25], we adopt the scaled tanh function starting with a smoothed objective function and becoming more non-smooth with the training process to attack the ill-posed gradient problem. The relationship between the sign function and the scaled tanh function can be defined as:

$$
\mathbf{B}=\lim _{\zeta \rightarrow \infty} \tanh (\zeta \mathbf{F})=\operatorname{sgn}(\mathbf{F})
$$

where $\zeta>0$ is a scaling parameter. We set $\zeta=1$ initially and increase $\zeta$ with the training process. When $\zeta \rightarrow \infty$, the network will converge to the original hash coding problem with sign activation function and yield exactly binary hash codes with the lowest quantization error. Algorithm 1 outlines the JDSH in details.

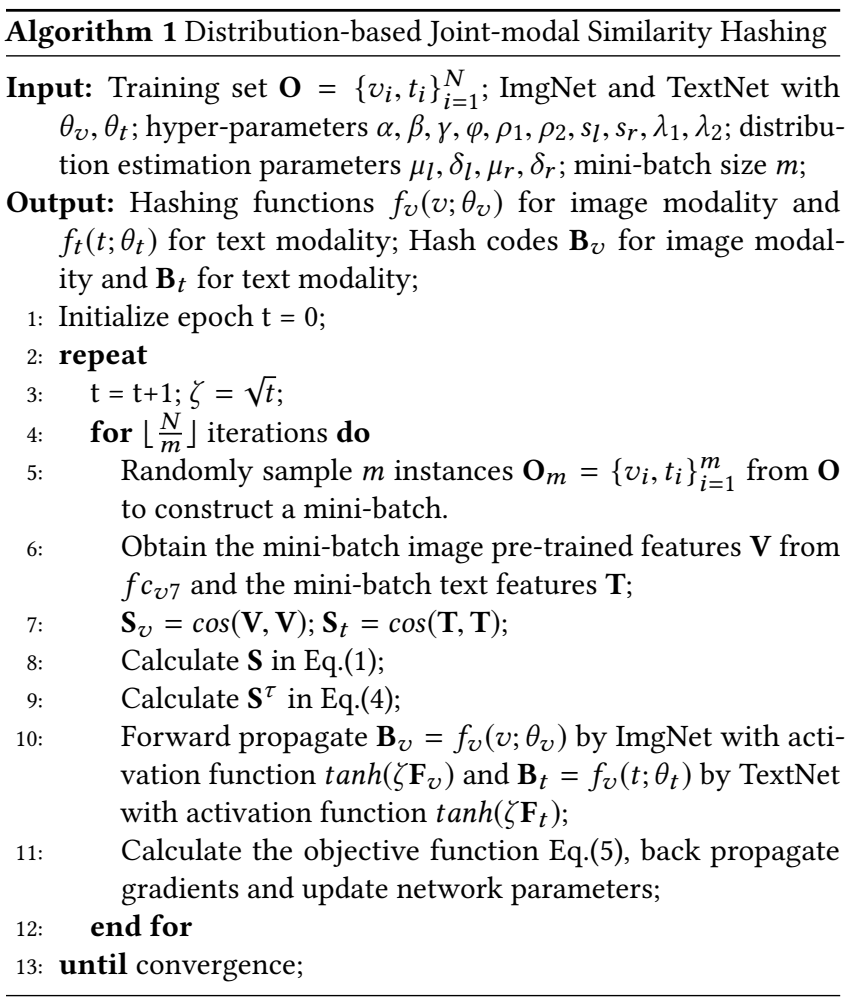

\section{EXPERIMENTS}

In this section, we conduct sufficient experiments on two datasets NUS-WIDE and MIRFlickr to verify the superiority of JDSH.

\subsection{Datasets}

NUS-WIDE [4] composes of 269,648 image-textual tag pairs in 81 concepts. Following [25, 33], 10 largest concepts are selected and 186,577 image-textual tag pairs are preserved. We randomly pick up 2,000 image-textual tag pairs as the query and the rest as the database (5,000 training pairs included). Each image is represented by 500 -dimensional SIFT feature and an index vector of the most frequent 1,000 textual tags is selected for each text.

MIRFlickr [13] consists of 25,000 image-text pairs annotated with 24 provided labels. Each image and text is represented by 100-dimensional SIFT descriptors and 500-dimensional tag vectors. Following the setting on [25,33], we randomly pick up 2,000 imagetext pairs as the query and the rest as the database (5,000 training pairs included).

\subsection{Baseline Methods and Evaluation Metrics}

Four unsupervised shadow cross-modal hashing methods $\mathrm{CVH}$ [17], IMH [24], CMFH [6] and LSSH [41], and three unsupervised deep cross-modal hashing methods DJSRH[25], UDCMH[33] and 
Table 1: The mAP@50 results of NUS-WIDE and MIRFlikcr at various code lengths.

\begin{tabular}{|c|c|c|c|c|c|c|c|c|c|}
\hline \multirow{2}{*}{ Task } & \multirow{2}{*}{ Methods } & \multicolumn{4}{|c|}{ NUS-WIDE } & \multicolumn{4}{|c|}{ MIRFlickr } \\
\hline & & 16bits & 32bits & 64bits & 128bits & 16bits & 32bits & 64bits & 128bits \\
\hline \multirow{8}{*}{$I \rightarrow T$} & CVH [17] & 0.372 & 0.363 & 0.404 & 0.390 & 0.606 & 0.599 & 0.596 & 0.589 \\
\hline & IMH [24] & 0.470 & 0.473 & 0.476 & 0.459 & 0.612 & 0.601 & 0.592 & 0.579 \\
\hline & CMFH [6] & 0.455 & 0.459 & 0.465 & 0.467 & 0.621 & 0.624 & 0.625 & 0.627 \\
\hline & LSSH [41] & 0.481 & 0.489 & 0.507 & 0.507 & 0.584 & 0.599 & 0.602 & 0.614 \\
\hline & DBRC [5] & 0.424 & 0.459 & 0.447 & 0.447 & 0.617 & 0.619 & 0.620 & 0.621 \\
\hline & UDCMH [33] & 0.511 & 0.519 & 0.524 & 0.558 & 0.689 & 0.698 & 0.714 & 0.717 \\
\hline & DJSRH [25] & 0.724 & 0.773 & 0.798 & 0.817 & 0.810 & 0.843 & 0.862 & 0.876 \\
\hline & JDSH & 0.736 & 0.793 & 0.832 & 0.835 & 0.832 & 0.853 & 0.882 & 0.892 \\
\hline \multirow{8}{*}{$T \rightarrow I$} & 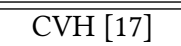 & 0.401 & $\overline{0.384}$ & 0.442 & 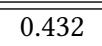 & 0.591 & 0.583 & $\overline{0.576}$ & $\overline{0.576}$ \\
\hline & IMH [24] & 0.478 & 0.483 & 0.472 & 0.462 & 0.603 & 0.595 & 0.589 & 0.580 \\
\hline & CMFH [6] & 0.529 & 0.577 & 0.614 & 0.645 & 0.642 & 0.662 & 0.676 & 0.685 \\
\hline & LSSH [41] & 0.577 & 0.617 & 0.642 & 0.663 & 0.637 & 0.659 & 0.659 & 0.672 \\
\hline & DBRC [5] & 0.455 & 0.459 & 0.468 & 0.473 & 0.618 & 0.626 & 0.626 & 0.628 \\
\hline & UDCMH [33] & 0.637 & 0.653 & 0.695 & 0.716 & 0.692 & 0.704 & 0.718 & 0.733 \\
\hline & DJSRH [25] & 0.712 & 0.744 & 0.771 & 0.789 & 0.786 & 0.822 & 0.835 & 0.847 \\
\hline & JDSH & 0.721 & 0.795 & 0.794 & 0.804 & 0.825 & 0.864 & 0.878 & 0.880 \\
\hline
\end{tabular}

DBRC[5] are selected as the baseline methods to compare with JDSH.

We adopt two standard retrieval evaluation metrics: mean Average Precision (mAP) and topN-precision curve to evaluate the retrieval performance of all methods. MAP is one of the most commonly-used metrics to jointly evaluate search accuracy and rankings. Given a query and a list of $R$ retrieval results, the average precision (AP) of the query is defined as:

$$
A P=\frac{1}{n} \sum_{r=1}^{R} P(r) \delta(r)
$$

where $n$ is the number of ground-truth similar instances of the query in the database, and $P(r)$ refers to the precision of the top $r$ retrieved instances. $\delta(r)=1$ when the $r$-th retrieval instance is similar to the query, otherwise $\delta(r)=0 . \mathrm{mAP}$ is defined as the average of APs for all queries. We follow [5, 25, 33] to set $R=50$ in our experiments. The topN-precision denotes the precision at different numbers of retrieved instances.

\subsection{Implementation Details}

We use mini-batch SGD optimizer with 0.9 momentum and 0.0005 weight decay to optimize the ImgNet and TextNet. The mini-batch size $m$ is 32 . The initial learning rates of ImgNet are 0.001 and 0.01 for NUS-WIDE and MIRFlickr respectively. Note that we only optimize the hashing layer of ImgNet (other layers are frozen) for MIRFlickr. The initial learning rates of TextNet are both 0.01 for two datasets.

The selection of hyper-parameters. First, we cross-validate the hyper-parameters $\alpha, \beta, \gamma, \varphi, \rho_{1}, \rho_{2}$ and finally set $\alpha=0.4, \beta=$ $0.3, \gamma=0.3, \varphi=1.5, \rho_{1}=0.3, \rho_{2}=0.3$ for NUS-WIDE and $\alpha=$ $0.5, \beta=0.1, \gamma=0.4, \varphi=1.47, \rho_{1}=0.1, \rho_{2}=0.1$ for MIRFlikcr. Second, we analyze the statistics of $\mathbf{S}=\left\{s_{i j}\right\}_{i, j=1}^{N}$ and then fit the left part of Gaussian distribution and the right part of Laplace distribution. Third, we cross-validate the hyper-parameters $\eta_{l}$, $\eta_{r}, \lambda_{1}$ and $\lambda_{2}$ in the distribution decision method and finally take $\eta_{l}=2, \eta_{r}=4, \lambda_{1}=0.2, \lambda_{2}=0.2$ for NUS-WIDE and $\eta_{l}=2, \eta_{r}=$ $4, \lambda_{1}=0.1, \lambda_{2}=0.2$ for MIRFlickr.

In addition, we follow [25] to preprocess $\mathbf{S}_{v}$ and $\mathbf{S}_{t}$ with $\mathbf{S}_{v}=$ $2 \mathbf{S}_{v}-1$ and $\mathbf{S}_{t}=2 \mathbf{S}_{t}-1$, respectively. Because the $\mathbf{F}_{v}, \mathbf{F}_{t}$ are the nonnegative representation which can cause $\mathbf{S}_{v}, \mathbf{S}_{t} \in[0,1]^{N \times N}$, the preprocessing transforms $\mathbf{S}_{v}, \mathbf{S}_{t}$ to $[-1,1]^{N \times N}$.

\subsection{Results and Discussions}

Table 1 shows the mAP results of JDSH and other comparison methods on NUS-WIDE and MIRFlickr in two cross-modal retrieval tasks for four lengths of hash code. $I \rightarrow T$ refers that the query is image modality and the database is text modality, and $T \rightarrow I$ refers that the query is text modality and the database is image modality. As shown in the results, JDSH outperforms all comparison methods. Especially, compared to the best unsupervised shadow cross-modal hashing methods LSSH, we achieve boosts of $29.7 \%$ and $15.38 \%$ in average $\mathrm{mAP}$ for different hash code lengths in $I \rightarrow T$ task and $T \rightarrow I$ task on NUS-WIDE respectively and achieve boosts of $25.95 \%$ and $20.55 \%$ in two retrieval tasks on MIRFlickr. Compared to DJSRH, the state-of-the-art unsupervised deep cross-modal hashing method, our method achieves increases of $2.5 \%$ and $2.45 \%$ in average mAP with different hash code lengths in $I \rightarrow T$ task and $T \rightarrow I$ task on NUS-WIDE respectively and achieves increases of $1.15 \%$ and $3.85 \%$ in two retrieval tasks on MIRFlickr.

Figure 4 shows the precision@top-N curves among all comparison methods. As shown in the figures, JDSH also outperforms all other comparison methods significantly which demonstrates JDSH can achieve higher retrieval precision in cross-modal retrieval and capture correlations between instances accurately.

The above experimental results verify the superiority of JDSH and indicate that JDSH can achieve better cross-modal retrieval performance and bridge modality gap better than other comparison methods. 


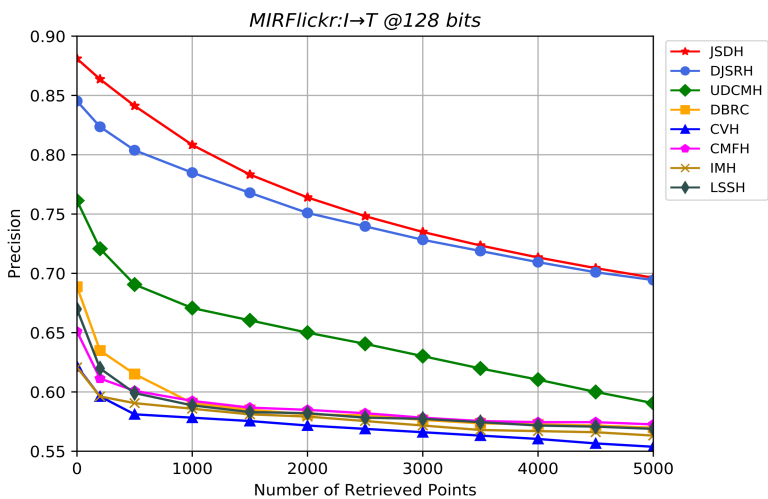

(a)

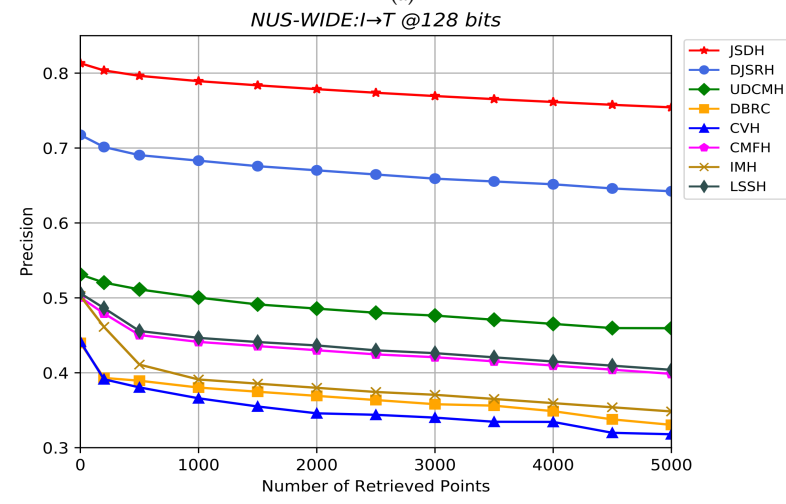

(c)

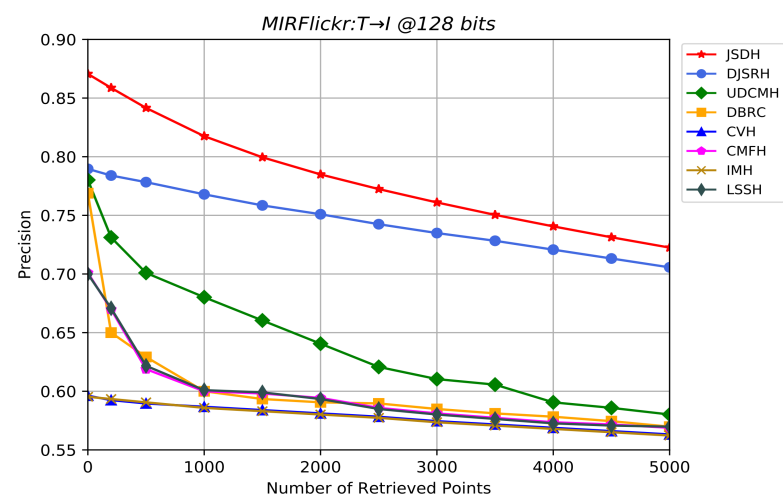

(b)

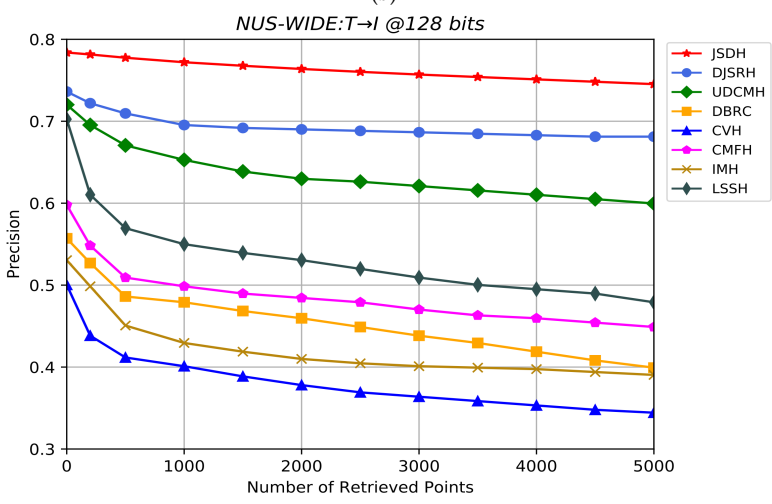

(d)

Figure 4: The topN-curves at 128 bits.

\subsection{Compare to State-of-the-art}

DJSRH also integrates cross-modal similarity information in a jointmodal semantic similarity matrix to supervise the hash coding process. But they obtain $\mathbf{S}^{\tau}$ by this manner: $\mathbf{S}^{\tau}=(1-\beta) \mathbf{S}+\beta \frac{\mathbf{S} \mathbf{S}^{T}}{m}$, where $\mathbf{S}=\alpha \mathbf{S}_{v}+(1-\alpha) \mathbf{S}_{t}, \alpha, \beta \in[0,1]$ and $m$ is the batch size. The experimental results demonstrate that our cross-modal similarity information integration manner described in Eq.(4) and Eq.(5) can capture the latent intrinsic semantics similarity better and have larger semantic representation capability than that of higher order fusion manner in DJSRH.

Furthermore, DSDW method improves the sampling and weighting scheme for unsupervised cross-modal hashing methods and generates more discriminative hash codes which further improve the cross-modal retrieval performance.

\subsection{Ablation Study}

We design several variants to verify the impacts of the proposed components and demonstrate the superiority of original JDSH. The proposed components that are needed to be verified are listed as follows:

- The joint-modal similarity matrix described in Eq.(1).

- DSDW method described in Eq.(4).

- The effective of intra-modal reconstruction described in Eq.(5).
Table 2: The mAP@50 results of four types of JDSH at 64 and 128 bits on MIRFlickr

\begin{tabular}{|c|c|c|c|c|c|}
\hline \multirow{2}{*}{ Method } & \multirow{2}{*}{ Configuration } & \multicolumn{2}{|c|}{64 bits } & \multicolumn{2}{|c|}{128 bits } \\
\hline & & $I \rightarrow T$ & $T \rightarrow I$ & $I \rightarrow T$ & $T \rightarrow I$ \\
\hline JDSH-1 & $\begin{array}{c}\text { w/o DSDW } \\
\mathbf{S}^{\tau}=\mathbf{S}=\alpha \mathbf{S}_{v}+(1-\alpha) \mathbf{S}_{t} \\
\text { with intra-reconstruction }\end{array}$ & 0.825 & 0.823 & 0.837 & 0.832 \\
\hline JDSH-2 & $\begin{array}{c}\text { w/o DSDW } \\
\mathbf{S}^{\tau}=\mathbf{S} \text { in Eq.(4) } \\
\text { with intra-reconstruction }\end{array}$ & 0.872 & 0.870 & 0.883 & 0.867 \\
\hline JDSH-3 & $\begin{array}{c}\text { with DSDW } \\
\mathbf{S}^{\tau} \text { in Eq.(4) (5) } \\
\text { w/o intra-reconstruction }\end{array}$ & 0.876 & 0.868 & 0.885 & 0.870 \\
\hline JDSH & $\begin{array}{c}\text { with DSDW and } \\
\mathbf{S}^{\tau} \text { in Eq.(4) (5) } \\
\text { with intra-reconstruction }\end{array}$ & 0.882 & 0.878 & 0.892 & 0.880 \\
\hline
\end{tabular}

The variants that are used to verify the impacts of above components are designed as follows:

- JDSH-1 abandons DSDW and adopts $\mathbf{S}^{\tau}=\mathbf{S}=\alpha \mathbf{S}_{v}+(1-$ $\alpha) \mathbf{S}_{t}$ as the final similarity matrix. The intra-modal reconstruction is included. 
- JDSH-2 abandons DSDW method and adopts $\mathbf{S}^{\tau}=\mathbf{S}$ described in Eq.(4) as the final similarity matrix. The intramodal reconstruction is included.

- JDSH-3 includes DSDW method and adopts $\mathbf{S}^{\tau}=\mathbf{S}$ described in Eq.(4) as the similarity matrix. The intra-modal reconstruction is abandoned.

We conduct $I \rightarrow T$ and $T \rightarrow I$ tasks at 64 and 128 bits on MIRFlickr dataset and the mAP@50 results of four types of JDSH are shown in Table 2. From the results, we have the following observations:

(1) From the mAP results of JDSH-1 and JDSH-2, we find that the performance improvement of JDSH-2 owes to the way of cross-modal similarity information fusion manner $\cos \left(\mathbf{S}_{v}, \mathbf{S}_{t}\right)$ in which the significant modality similarity information can be fused effectively. Meanwhile, this manner is helpful to bridge the modality gap.

(2) From the results of JDSH and JDSH-2, the performance improvement of JDSH attributes to DSDW method which is effective to generate more discriminative and accurate hash codes by improving the sampling and weighting schemes.

(3) The mAP results of JDSH and JDSH-3 demonstrate that the intra-modal reconstruction is effective to improve the retrieval performance by considering the intra-modal retrieval situation.

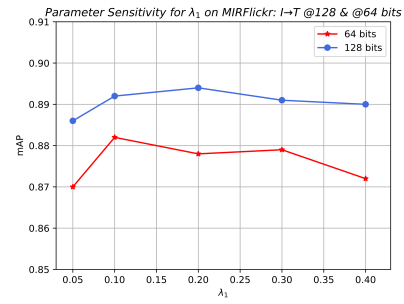

(a)

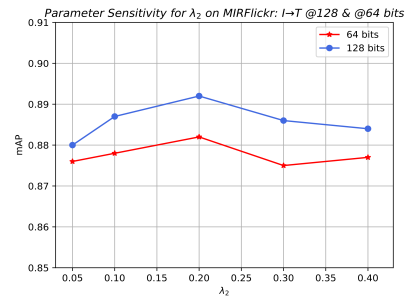

(c)

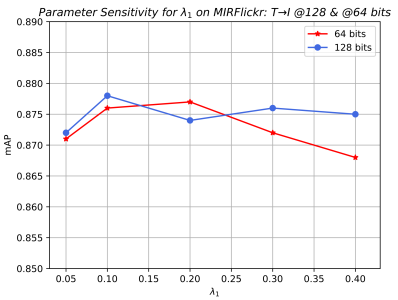

(b)

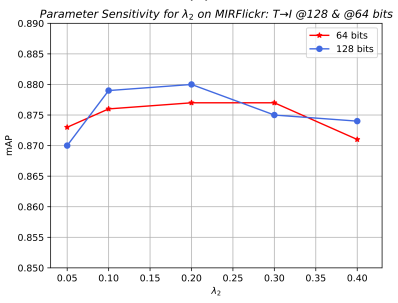

(d)
Figure 5: Parameter Sensitivity for $\lambda_{1}$ and $\lambda_{2}$.

\subsection{Parameter Sensitivity}

In this section, we analyse the hyper-parameters of the Distributionbased Similarity Decision and Weighting method in terms of sensitivity on dataset MIRFlickr, including $\eta_{l}, \eta_{r}, \lambda_{1}$ and $\lambda_{2}$.

First, we explore the sensitivity of hyper-parameters $\lambda_{1}$ and $\lambda_{2}$ by fixing $\eta_{l}, \eta_{r}$ as 2,4 respectively. The effect of $\lambda_{1}$ and $\lambda_{2}$ in DSDW on dataset MIRFlickr for $I \rightarrow T$ and $T \rightarrow I$ tasks with hash code lengths of 64 and 128 bits is shown in Figure 5. In order to investigate the sensitivity of $\lambda_{1}$, we fix $\lambda_{2}$ as 0.2 and vary $\lambda_{1}$ from 0.05 to 0.4 to evaluate the mAP results. The Figure 5(a) and Figure 5 (b) present the results and we obtain the best results when $\lambda_{1}$ is

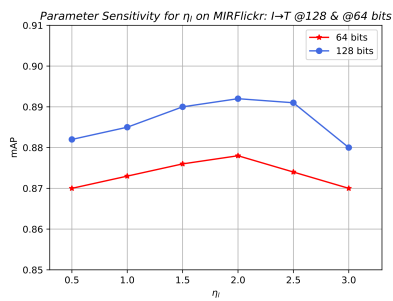

(a)

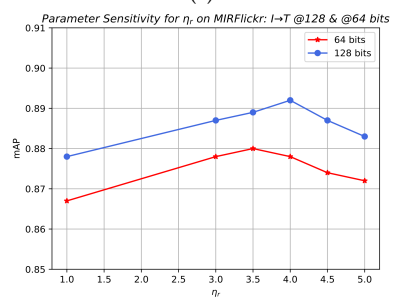

(c)

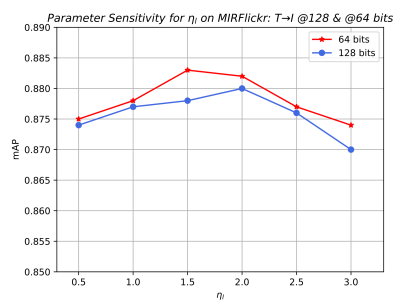

(b)

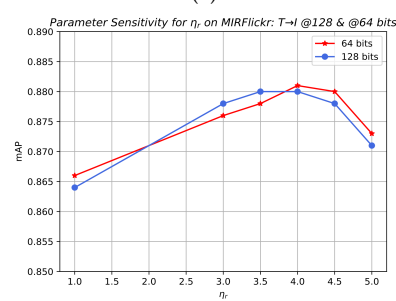

(d)
Figure 6: Parameter Sensitivity for $\eta_{l}$ and $\eta_{r}$.

set to 0.1 . Then we fix $\lambda_{1}$ to 0.1 and vary $\lambda_{2}$ from 0.05 to 0.4 to evaluate the mAP results. The results are presented in Figure 5(c) and Figure 5(d), and the best results are achieved when $\lambda_{2}$ is 0.2 . Thus, we select $\lambda_{1}, \lambda_{2}$ as $0.1,0.2$ on dataset MIRFlickr, respectively.

Second, we fix $\lambda_{1}, \lambda_{2}$ as $0.1,0.2$ respectively and explore the sensitivity of hyper-parameters $\eta_{l}$ and $\eta_{r}$ that control the similarity thresholds in DSDW. Figure 6 shows the effect of $\eta_{l}$ and $\eta_{r}$ in DSDW on dataset MIRFlickr for $I \rightarrow T$ and $T \rightarrow I$ tasks with hash code lengths of 64 and 128 bits. In order to explore the sensitivity of $\eta_{l}$, we fix $\eta_{r}$ as 4 and vary $\eta_{l}$ from 0.5 to 3.5 to evaluate the mAP results. The Figure 6(a) and Figure 6(b) present the results and we achieve the best results when $\eta_{l}$ is set to 2 . Then we fix $\eta_{l}$ as 2 and vary $\eta_{r}$ from 1 to 4 to evaluate the mAP results. The results are presented in Figure 5(c) and Figure 5(d), and the best results are achieved when $\eta_{r}$ is 4 . Especially, the mAP results drop dramatically when $\eta_{r}$ is set to 1 . This is because the value of $\eta_{r}$ is set too small, so that too many instance pairs are considered as semantic similar pairs and that the similarity between them is mistakenly increased by DSDW. Finally, we select $\eta_{l}, \eta_{r}$ as 2, 4 respectively on dataset MIRFlickr.

\section{CONCLUSION AND FUTURE WORK}

In this paper, we propose a novel Joint-modal Distribution-based Similarity Hashing (JDSH) for large-scale cross-modal retrieval. In JDSH, for better preserving similarity information from different modalities and bridging modality gap, we introduce a novel crossmodal joint-training method by constructing a joint-modal similarity matrix which is able to fully preserve the cross-modal semantic correlations among instances and mine the latent intrinsic modality characteristics. For improving the sampling and weighting schemes of unsupervised cross-modal hashing, the Distribution-based Similarity Decision and Weighting (DSDW) method is proposed to generate more discriminative and accurate hash codes. We first define four types of similarities to exactly describe the relationships among instances in unsupervised pair-based problems. We then analyze the statistics of the joint-modal similarity matrix and find that the similarity distribution can be estimated by a left half 
Gaussian distribution and a right half Laplace distribution. According to the estimated distributions, we set two similarity thresholds and impose the designed weighting scheme to selected instances that makes similar instances closer and dissimilar instances apart. Extensive experiments have demonstrated that JDSH can achieve better cross-modal retrieval performances compared with recent unsupervised deep cross-modal hashing methods.

The proposed DSDW is an initial sampling and weighting scheme for unsupervised cross-modal retrieval. In the future, the performance of DSDW method in other unsupervised pair-based problems will be further explored. Furthermore, we will further investigate a more effective and advanced paradigm to enhance feature representation from different modalities and bridge modality gap better, e.g. Graph Convolutional Network [15].

\section{ACKNOWLEDGMENTS}

This work was supported in part by the National Natural Science Foundation of China under Grants 61802405.

\section{REFERENCES}

[1] Yue Cao, Mingsheng Long, Jianmin Wang, Qiang Yang, and Philip S Yu. 2016. Deep visual-semantic hashing for cross-modal retrieval. In SIGKDD. ACM, 14451454 .

[2] Yue Cao, Mingsheng Long, Jianmin Wang, and Philip S Yu. 2016. Correlation hashing network for efficient cross-modal retrieval. BMVC (2016).

[3] Zhangjie Cao, Mingsheng Long, Jianmin Wang, and Philip S Yu. 2017. Hashnet Deep learning to hash by continuation. In ICCV. 5608-5617.

[4] Tat-Seng Chua, Jinhui Tang, Richang Hong, Haojie Li, Zhiping Luo, and Yantao Zheng. 2009. NUS-WIDE: a real-world web image database from National University of Singapore. In ICIVR. 48-56.

[5] Feiping Nie Di Hu and Xuelong Li. 2018. Deep Binary Reconstruction for Crossmodal Hashing. IEEE Transactions on Multimedia (2018), 973-985.

[6] Guiguang Ding, Yuchen Guo, Jile Zhou, and Yue Gao. 2016. Large-scale crossmodality search via collective matrix factorization hashing. IEEE Transactions on Image Processing 25, 11 (2016), 5427-5440.

[7] Ross Girshick, Jeff Donahue, Trevor Darrell, and Jitendra Malik. 2014. Rich feature hierarchies for accurate object detection and semantic segmentation. In CVPR. 580-587.

[8] Wen Gu, Xiaoyan Gu, Jingzi Gu, Bo Li, Zhi Xiong, and Weiping Wang. 2019 Adversary Guided Asymmetric Hashing for Cross-Modal Retrieval. In ICMR. ACM, 159-167.

[9] Raia Hadsell, Sumit Chopra, and Yann LeCun. 2006. Dimensionality reduction by learning an invariant mapping. In CVPR, Vol. 2. IEEE, 1735-1742.

[10] Kaiming He, Xiangyu Zhang, Shaoqing Ren, and Jian Sun. 2016. Deep residual learning for image recognition. In CVPR. 770-778.

[11] Elad Hoffer and Nir Ailon. 2015. Deep metric learning using triplet network. In International Workshop on Similarity-Based Pattern Recognition. Springer, 84-92.

[12] Gao Huang, Zhuang Liu, Laurens Van Der Maaten, and Kilian Q Weinberger 2017. Densely connected convolutional networks. In CVPR. 4700-4708.

[13] Mark J. Huiskes, Bart Thomee, and Michael S. Lew. 2008. The MIR flickr retrieval evaluation initiative. In ICMIR. 39-43.

[14] Qing-Yuan Jiang and Wu-Jun Li. 2017. Deep cross-modal hashing. In CVPR. 3232-3240.

[15] Thomas N Kipf and Max Welling. 2016. Semi-supervised classification with graph convolutional networks. arXiv preprint arXiv:1609.02907 (2016).

[16] Alex Krizhevsky, Ilya Sutskever, and Geoffrey E. Hinton. 2012. ImageNet Classification with Deep Convolutional Neural Networks. In NIPS. 1097-1105.

[17] Shaishav Kumar and Raghavendra Udupa. 2011. Learning hash functions for cross-view similarity search. In IFCAI. 1360-1365.

[18] Chao Li, Cheng Deng, Ning Li, Wei Liu, Xinbo Gao, and Dacheng Tao. 2018 Self-Supervised Adversarial Hashing Networks for Cross-Modal Retrieval. In CVPR. 4242-4251.

[19] Zijia Lin, Guiguang Ding, Mingqing Hu, and Jianmin Wang. 2015. Semanticspreserving hashing for cross-view retrieval. In CVPR. 3864-3872.

[20] Xu Lu, Lei Zhu, Zhiyong Cheng, Jingjing Li, Xiushan Nie, and Huaxiang Zhang 2019. Flexible Online Multi-modal Hashing for Large-scale Multimedia Retrieval. In ACM MM. 1129-1137.

[21] Hyun Oh Song, Yu Xiang, Stefanie Jegelka, and Silvio Savarese. 2016. Deep metric learning via lifted structured feature embedding. In CVPR. 4004-4012.
[22] David E Rumelhart, Geoffrey E Hinton, James L McClelland, et al. 1986. A general framework for parallel distributed processing. Parallel distributed processing: Explorations in the microstructure of cognition 1, 45-76 (1986), 26.

[23] Karen Simonyan and Andrew Zisserman. 2014. Very deep convolutional networks for large-scale image recognition. arXiv preprint arXiv:1409.1556 (2014).

[24] Jingkuan Song, Yang Yang, Yi Yang, Zi Huang, and Heng Tao Shen. 2013. Intermedia hashing for large-scale retrieval from heterogeneous data sources. In SIGMOD. ACM, 785-796.

[25] Shupeng Su, Zhisheng Zhong, and Chao Zhang. 2019. Deep Joint-Semantics Reconstructing Hashing for Large-Scale Unsupervised Cross-Modal Retrieval. In ICCV.

[26] Christian Szegedy, Sergey Ioffe, Vincent Vanhoucke, and Alexander A Alemi. 2017. Inception-v4, inception-resnet and the impact of residual connections on learning. In $A A A I$.

[27] Christian Szegedy, Vincent Vanhoucke, Sergey Ioffe, Jon Shlens, and Zbigniew Wojna. 2016. Rethinking the inception architecture for computer vision. In CVPR. 2818-2826.

[28] Bokun Wang, Yang Yang, Xing Xu, Alan Hanjalic, and Heng Tao Shen. 2017. Adversarial cross-modal retrieval. In ACM MM. 154-162.

[29] Jian Wang, Feng Zhou, Shilei Wen, Xiao Liu, and Yuanqing Lin. 2017. Deep metric learning with angular loss. In ICCV. 2593-2601.

[30] Xun Wang, Xintong Han, Weilin Huang, Dengke Dong, and Matthew R Scott. 2019. Multi-Similarity Loss with General Pair Weighting for Deep Metric Learning. In CVPR. 5022-5030.

[31] Yair Weiss, Antonio Torralba, and Rob Fergus. 2009. Spectral hashing. In NeurIPS. 1753-1760.

[32] Dayan Wu, Qi Dai, Jing Liu, Bo Li, and Weiping Wang. 2019. Deep Incremental Hashing Network for Efficient Image Retrieval. In CVPR. 9069-9077.

[33] Gengshen Wu, Zijia Lin, Jungong Han, Li Liu, Guiguang Ding, Baochang Zhang, and Jialie Shen. 2018. Unsupervised Deep Hashing via Binary Latent Factor Models for Large-scale Cross-modal Retrieval.. In IfCAI. 2854-2860.

[34] Ruiqing Xu, Chao Li, Junchi Yan, Cheng Deng, and Xianglong Liu. 2019. Graph convolutional network hashing for cross-modal retrieval. In IFCAI. 10-16.

[35] Erkun Yang, Cheng Deng, Tongliang Liu, Wei Liu, and Dacheng Tao. 2018. Semantic Structure-based Unsupervised Deep Hashing. In IFCAI. 1064-1070.

[36] Erkun Yang, Cheng Deng, Wei Liu, Xianglong Liu, Dacheng Tao, and Xinbo Gao. 2017. Pairwise relationship guided deep hashing for cross-modal retrieval. In $A A A I$.

[37] Erkun Yang, Tongliang Liu, Cheng Deng, Wei Liu, and Dacheng Tao. 2019. DistillHash: Unsupervised Deep Hashing by Distilling Data Pairs. In CVPR. 2946-2955.

[38] Dong Yi, Zhen Lei, Shengcai Liao, and Stan Z Li. 2014. Deep metric learning for person re-identification. In ICPR. IEEE, 34-39.

[39] Dongqing Zhang and Wu-Jun Li. 2014. Large-scale supervised multimodal hashing with semantic correlation maximization. In $A A A I$

[40] Liangli Zhen, Peng Hu, Xu Wang, and Dezhong Peng. 2019. Deep Supervised Cross-Modal Retrieval. In CVPR. 10394-10403.

[41] Jile Zhou, Guiguang Ding, and Yuchen Guo. 2014. Latent semantic sparse hashing for cross-modal similarity search. In SIGIR. ACM, 415-424. 